\title{
Simulations of copper single crystals subjected to rapid shear
}

\author{
Andrew Higginbotham, ${ }^{1, a)}$ Eduardo M. Bringa, ${ }^{2}$ Jaime Marian, ${ }^{3}$ Nigel Park, ${ }^{4}$ \\ Matthew Suggit, ${ }^{1}$ and Justin S. Wark ${ }^{1}$ \\ ${ }^{1}$ Department of Physics, Clarendon Laboratory, University of Oxford, Parks Road, Oxford OXI 3PU, United \\ Kingdom \\ ${ }^{2}$ CONICET \& Instituto de Ciencias Básicas, Universidad Nacional de Cuyo, Mendoza, 5500 Argentina \\ ${ }^{3}$ Physical and Life Sciences Directorate, Lawrence Livermore National Laboratory, Livermore, California \\ 94551, USA \\ ${ }^{4}$ AWE, Aldermaston, Reading RG7 4PR, United Kingdom
}

(Received 28 June 2010; accepted 31 January 2011; published online 00 March 2011)

\begin{abstract}
We report on nonequilibrium molecular dynamics simulations of single crystals of copper experiencing rapid shear strain. A model system, with periodic boundary conditions, which includes a single dislocation dipole is subjected to a total shear strain of close to $10 \%$ on time-scales ranging from the instantaneous to $50 \mathrm{ps}$. When the system is strained on a time-scale short compared with a phonon period, the initial total applied shear is purely elastic, and the eventual temperature rise in the system due to the subsequent plastic work can be determined from the initial elastic strain energy. The rate at which this plastic work occurs, and heat is generated, depends on the dislocation velocity, which itself is a function of shear stress. A determination of the stress-dependence of the dislocation velocity allows us to construct a simple analytic model for the temperature rise in the system as a function of strain rate, and this model is found to be in good agreement with the simulations. For the effective dislocation density within the simulations, $7.8 \times 10^{11} \mathrm{~cm}^{-2}$, we find that applying the total shear strain on time-scales of a few tens of picoseconds greatly reduces the final temperature. We discuss these results in the context of the growing interest in producing high pressure, solid-state matter, by quasi-isentropic (rather than shock) compression. (C) 2011 American Institute of Physics. [doi:10.1063/1.3560912]
\end{abstract}

\section{INTRODUCTION}

The dissipation of heat due to plastic work is one of the fundamental phenomena exhibited when materials are deformed beyond their elastic limits, and has been the subject of study ever since the discovery that the creation and motion of dislocations and defects is the means by which plastic work is generally dissipated. ${ }^{1-4}$ Plastic heating can be particularly high in materials subjected to rapid compression, such as that found within the environment of a shock. Indeed, the rapid temperature rise that occurs across the shock front eventually leads to shock-melting if the compression is sufficiently great. The pressure at which such melting occurs on the Hugoniot is clearly material dependent, but for all but the stiffest materials a pressure of 1-2 Mbar is typical. ${ }^{5}$ Notably, this pressure lies a little below the highest pressures that can be achieved statically in the laboratory by use of diamond anvil cells (DACs). ${ }^{6}$ As the melting noted above places an upper limit on the pressure to which a material can be shocked, and yet still remain solid, in recent years there has been a growing interest in the development of techniques which allow materials to be compressed dynamically, and indeed rapidly, but not so rapidly as to produce a shock: such techniques generally being termed 'quasi-isentropic' compression. $^{7-10}$ The rationale for such experiments is that dynamic compression methods (such as laser driven compression, or magnetic loading using Z-pinches), can reach

\footnotetext{
a) Author to whom correspondence should be addressed. Electronic mail: andrew.higginbotham@physics.ox.ac.uk.
}

peak pressures far in excess of those achievable with current DACs, and if the time-dependence of the applied stress can be controlled, such approaches may allow the creation, albeit transiently, of solid-state matter in regions of the phase diagram that have hitherto remained unexplored. It has been proposed that diagnosis of the state of the material produced could be performed using flash x-ray diffraction, as such techniques have been shown to be successful in obtaining structural information on materials subjected to shock compression. ${ }^{16}$ Some significant successes have already been achieved in this field, with recent studies employing ramped compression reporting diamond which is still solid at 8 Mbar. ${ }^{17}$ These ramped compression techniques are termed 'quasi-isentropic' as some plastic work must still be dissipated within the material: most of the techniques suggested and employed to date have relied upon uniaxial compression of the sample, and as the material will be subjected to stresses far beyond its elastic limit, plastic flow will certainly still take place. However, the underlying assumption is that the plastic work required will be considerably less than that which is required within the shock-compression scenario. It is in this context that we present the work outlined in this paper, where we explore by use of molecular dynamics (MD) simulations a simple model system, with fixed dislocation density, subjected to shear strain across a range of strain rates. Previous MD simulations of plastic flow have largely concentrated either on shock compression ${ }^{18-21}$ or on the steady state properties of plasticity; typically then being run at constant temperature and shear stress. ${ }^{22-29}$ Although this has produced a wealth of information used to inform multi-scale modeling 
approaches, it has left the field of plastic heating during high strain rate ramped compression (relevant to quasi-isentropic compression) largely unexplored.

Plastic flow has also been studied by dislocation dynamics (DD) techniques. ${ }^{30,31}$ In most DD simulations, the final strain is relatively small and the strain rates are much smaller than those found in shocks. Therefore, plastic heating is typically and rightfully neglected. In a recent development, a DD approach was combined with finite element methods (FEM) to simulate shock evolution. ${ }^{4}$ Here heating was assumed to result from a constant compliance and allowed to flow into the FEM mesh. This method was used to study relaxation under different ramp loadings. In the DD-FEM simulations, dislocation density was lower than in equivalent MD simulations, resulting in less pinning and enhanced dislocation motion. As a result, shear stress relaxation occurred faster, and plastic heating (with effectiveness of 90\%) was somewhat higher than that seen in the MD simulations. ${ }^{32}$

Our goal in this paper can be considered relatively modest. In real physical systems subjected to ramp loading (i.e., dynamic, but less rapid than a shock), the increase in temperature will be due to a combination of factors. Firstly, the compression itself will induce a temperature rise, which exists even if the compression is purely isentropic (though we note that for many materials this will keep the sample well away from the melting point, as under isentropic compression $\left(T / \theta_{D}\right)$, where $\theta_{D}$ is the Debye temperature, reduces assuming realistic values of the Gruneisen parameter ${ }^{33}$ ). Secondly, the defect generation and motion will induce a temperature rise associated with the plastic work, as the shear stresses relax. Thus, we consider here a very simple system: single crystal copper containing a lone dislocation dipole (the overall dislocation density being determined by the fact that we use periodic boundary conditions). We subject it to volume-conserving shear (thus neglecting temperature rises owing to compression) to a given total shear strain (i.e., a given tilt of the simulation cell) at a variety of strain rates, and we monitor both the shear stress and temperature as a function of time. The system is simulated using nonequilibrium $\mathrm{MD}$, and is also described with a basic analytical model based on Orowan's equation. Excellent agreement between the MD simulations and the analytical model is found, as long as the stress-dependence of the dislocation velocity is taken into account. We show that the highest temperature rise of the system occurs for shear on a time-scale comparable to (or shorter than) that of a phonon period. As we employ periodic boundary conditions, the effective dislocation density within the simulations, $7.8 \times 10^{11} \mathrm{~cm}^{-2}$, is determined by the size of the simulation box. Such a density is not too dissimilar to those expected within rapid-compression environments, and we find that if shear strains of close to $10 \%$ are applied on time-scales of a few tens of picoseconds, the temperature rise is significantly reduced. The good agreement between the MD simulations and the analytical model presented indicates that it may be possible to make reasonable predictions of the degree of heating during ramped compression of simple metals.

\section{THEORY}

A complete theoretical description of plastic relaxation is highly challenging (even in fcc solids where plasticity is medi- ated almost exclusively by the flow of dislocations). There are relatively few models which consider plastic heating. ${ }^{3,17,34,35}$

In this work we start by examining the simple case of full dislocations of Burgers vector $\mathbf{b}=\frac{1}{2}[01 \overline{1}]$ in the (111) plane gliding in response to an applied shear strain. We shall work with a coordinate system such that [111] is along $z$ and $[01 \overline{1}]$ is along $x$. In this system the glide of the dislocation will act to relieve the $\sigma_{13}$ component of the stress. This simple quasione dimensional arrangement lends itself well to the simple Maxwellian model of a viscoelastic solid. ${ }^{36}$

We assume that the strain, and its time derivative, can be expressed as a sum of its elastic and plastic components -

$$
\frac{\mathrm{d} \varepsilon_{13}^{\mathrm{ext}}}{\mathrm{dt}}=\frac{\mathrm{d} \varepsilon_{13}^{\mathrm{e}}}{\mathrm{dt}}+\frac{\mathrm{d} \varepsilon_{13}^{\mathrm{p}}}{\mathrm{dt}}
$$

For the case of an external $\varepsilon_{13}$ shear strain being relieved by dislocation glide, as described above, we can use a single component of the compliance tensor to relate elastic stress and strain -

$$
\varepsilon_{13}^{e}=c \sigma_{13}
$$

The validity of linear elasticity in context of the high strains applied in this work will be confirmed in section III. For the plastic strain rate we use Orowan's equation which states, for constant dislocation density -

$$
\frac{\mathrm{d} \varepsilon^{p}}{\mathrm{dt}}=\rho b v(\sigma)
$$

where $\rho$ is the number density of mobile dislocations with Burgers vectors of magnitude $b$, and $v(\sigma)$ their stress-dependent velocity. In situations of real physical interest, such as shock or rapid ramp compression of materials to high pressures, the time dependence of the dislocation density may be highly complex, with homogeneous and heterogeneous nucleation, multiplication and pinning all affecting the mobile dislocation density. As outlined in the introduction, our aim in this work is not to attempt to simulate such a complex situation, but to gain some modest insight into some of the underlying physics by studying a model system.

Taken together Eqs. (1)-(3) allow us to express the external strain rate as

$$
\begin{gathered}
\frac{\mathrm{d} \varepsilon_{13}^{\mathrm{ext}}}{\mathrm{dt}}=c \frac{\mathrm{d} \sigma_{13}}{\mathrm{dt}}+\rho b v(\sigma) \\
\Rightarrow \sigma_{13}\left(t^{\prime}\right)=\int_{0}^{t^{\prime}} \frac{1}{c} \frac{\mathrm{d} \varepsilon_{13}^{\mathrm{ext}}}{\mathrm{dt}}-\frac{\rho b v\left(\sigma_{13}\right)}{c} \mathrm{~d} t .
\end{gathered}
$$

The temperature rise due to dislocation glide can be found by noting that for an elasto-plastic solid, assuming isotropic linear thermoelasticity with infinitesimal deformations, and a linear Fourier heat conduction law, the unidimensional energy balance equation can be written as -

$$
\frac{C}{V} \frac{\partial T}{\partial t}-k \frac{\partial^{2} T}{\partial x^{2}}=\beta \sigma_{13} \frac{\mathrm{d} \varepsilon^{p}}{\mathrm{~d} t}-\frac{\alpha T}{c} \varepsilon^{e},
$$

where the additive decomposition of strain into elastic and plastic parts is once again assumed. Here $T$ is absolute 
temperature, $C$ the heat capacity of the sample (taken to be $3 N k T$ in these classical simlations), $V$ its volume, $k$ the thermal conductivity, and $\alpha$ its thermal expansion coefficient. $\beta$ is an empirical parameter that represents the fraction of the rate of plastic work dissipated as heat. ${ }^{37}$

Under the adiabatic, isochoric conditions considered in this paper, and by once again assuming Orowan's equation to describe the plastic strain rate, this can be reduced to -

$$
\mathrm{d} T=\beta \frac{V \sigma_{13}(t) \rho b v \mathrm{~d} t}{C} .
$$

The value of $\beta$ is material and conditions dependent, but for rapid loading takes values very close to unity. ${ }^{37}$ Here we will assume a value of $\beta=1$ but note that $\beta$ could also be used as an adjustable parameter.

\section{SIMULATIONS}

\section{A. System setup}

In order to test the model proposed in Sec. II we compare its predictions with those of nonequilibrium molecular dynamics simulations. The MD simulation was performed using the LAMMPS package ${ }^{38}$ and comprised a box of size $20.4 \times 10.6 \times 12.5 \mathrm{~nm}(228960$ atoms $)$ of copper atoms, modeled using the embedded atom method interatomic potential by Mishin et al. $^{39}$ This box was oriented with crystallographic axes as defined in Sec. II. An edge dislocation dipole was introduced into the box by removing two half planes and allowing the sample to relax as described elsewhere, ${ }^{40}$ leading to a box with equivalent dislocation density $\rho=7.8$ $\times 10^{11} \mathrm{~cm}^{-2}$. Although relatively modest compared to densities expected to be present within shock-compressed copper, ${ }^{21,41}$ it is sufficiently low to allow us to neglect dislocation interactions. The dipole and the orientation of the simulation cell are shown in Fig. 1.

Relaxation of the sample was carried out in the microcanonical (constant NVE) ensemble, leading to a simulation cell with a low initial temperature (around $30 \mathrm{~K}$ ), and a finite pressure of $1.4 \mathrm{GPa}^{42}$

A linear shear strain ramp was applied, from $\epsilon_{13}=0$ to 0.098 , during a time $t_{\text {rise }}$. In the absence of plastic relaxation

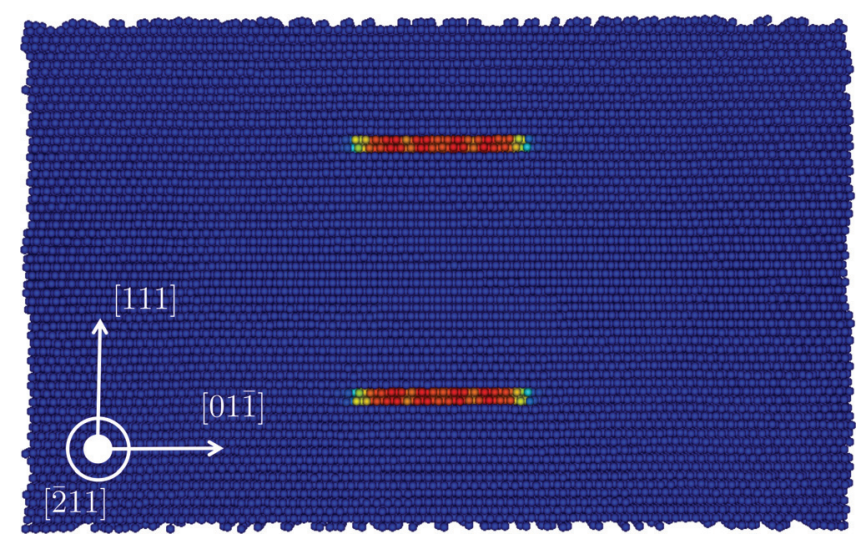

FIG. 1. (Color online) Simulation setup. Coloring with centrosymmetry parameter. The dislocation dipole can be seen immersed in the perfect crystal (blue), with partial dislocations in yellow and the stacking fault in red. Dislocation slip occurs along $[01 \overline{1}]$. this shear strain would lead to a peak shear stress in the sample of $4 \mathrm{GPa}$. This is a value typical of those which could be reached for shock pressures above $50 \mathrm{GPa}$. The sample was allowed to relax this applied strain both during and subsequent to the application of the ramp. Both the $\sigma_{13}$ stress component and the temperature were recorded as a function of time.

Over the range of strains investigated in this paper it was found that the stress-strain relation was linear, confirming our assumption of the validity of linear elasticity theory in this regime. ${ }^{43}$ The $c_{1313}$ compliance component of the sample was measured to be $0.025 \mathrm{GPa}^{-1}$.

\section{B. Dislocation velocity}

In order to solve Eq. (5), we require a knowledge of the dislocation velocity as a function of stress. One might assume, as a first approximation in this high stress, high strain rate regime, that the dislocation velocity has saturated and reached a constant value close to the Rayleigh speed (around $3660 \mathrm{~ms}^{-1}$ for $\mathrm{Cu}$ ). However, several previous MD simulation efforts have found that a linear stress dependence provides a better fit. ${ }^{22,23,25,44}$ One may choose to use the velocities reported in these steady state simulations as input to the model. We will use a simple fit to the data of Tsuzuki et al. for edge dislocation velocities in Mishin $\mathrm{Cu}^{26}$ However, we may consider these steady state dislocation mobilities to be inappropriate for the study of a highly dynamic process, especially considering that previous studies have shown dislocations can take over 10 ps to reach these steady state velocities; a time comparable to our dynamic studies. ${ }^{23}$

Therefore, in addition to fits to the steady state data, we also determine dislocation velocities from a dynamic simulation. We extract the velocity of the dislocations during relaxation from an instantaneous $\left(t_{\text {rise }}=0\right)$ shear. This is achieved by monitoring the 'center of mass' of the upper dislocation as a function of time, using the centrosymmetry parameter to identify the atoms in the defected environment. ${ }^{45}$ The resulting stress-velocity plot is shown in Fig. 2. The scatter in the data can be attributed to the small sample size and the difficulty in calculating average velocity over

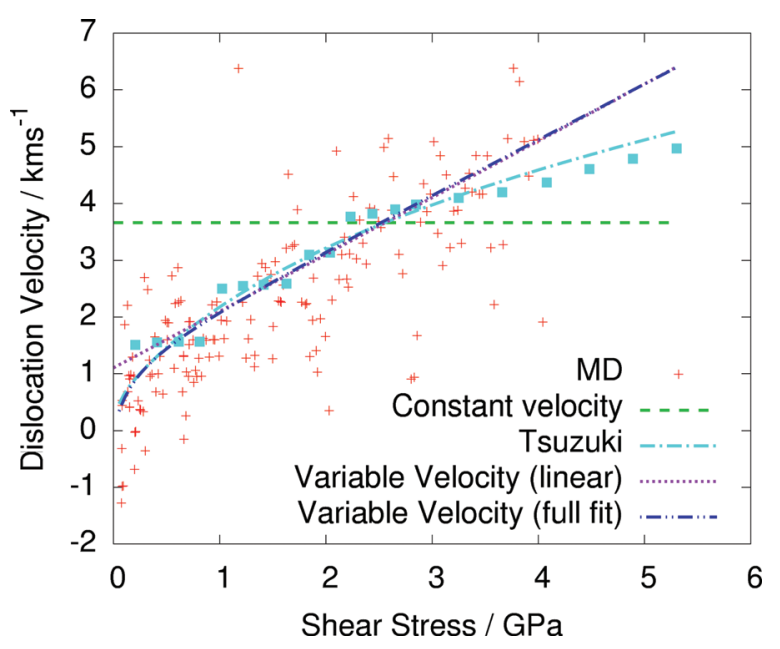

FIG. 2. (Color online) Dislocation velocity from our MD simulation and resulting linear and nonlinear fits. Steady state MD data reproduced from Tsuzuki et al. along with a fit to that data, is also shown. ${ }^{26}$ 
relatively short time spans. Note that this determination of velocity does not use the model described in Sec. II.

A simple constant velocity fit would seem to be inappropriate over this stress range. A linear fit to the data provides values in reasonable agreement with those found by Tsuzuki et al. over the mid-range of stress states. However, we see from Fig. 2 that as well as a slight divergence at high stress, this linear approximation will clearly break down for very low shear stresses (that may prevail at very low shear strain rates), and thus we also use a 'full' fit ensuring that the dislocation velocity tends to zero as the stress is reduced. The form of this full fit is the reciprocal sum of two linear functions chosen so as to agree with the linear fit at high stress (this form can be viewed as an approximation to the mobility model discussed by Olmsted ${ }^{46}$ ). However, most of the heating occurs for shear stresses in the high velocity regime, ${ }^{46}$ and thus we consider this a small correction. This fact also allows us to neglect the low velocity temperature dependence. This might not be the case if temperature approaches values closer to melting, where a temperature-dependent velocity would need to be incorporated in Eq. (7).

We will test both the steady state and dynamically determined velocities in the model of Sec. II.

\section{RESULTS}

First, we verify that the analytic model reproduces the shear stress relaxation due to dislocation motion in our simulations. Results for ramps of $t_{\text {rise }}=0,5,10$, and $20 \mathrm{ps}$ are shown in Fig. 3. One can consider the 0 ps case as being analogous to an ideal shock wave, with larger rise times representing ramps of decreasing shear strain rate.

For all but the lowest strain rate, it can be seen that the linear velocity, Tsuzuki, and full fit predictions are essentially indistinguishable. The agreement between the analytic model employing variable velocity of the dislocations and the results of the MD simulations is excellent, with only minor deviations present, regardless of whether dynamic or steady state dislocation velocities are used. For ramp times longer than 20 ps we see deviations from the predicted stress relief profiles, likely due to stress field fluctuations and dislocation inertia; phenomena absent in the analytic model. It should be noted that, as expected, longer ramps allow relaxation of the shear strain at lower shear stresses, and as shown in Eq. (7), this allows for less heat generation during relaxation of the shear strain.

The temperature rise as a function of time during the plastic heating is shown in Fig. 4. Once again, employing a variable velocity fit in the model proposed in Sec. II, we find a good agreement with the MD simulations. Note that the temperature rise (as defined by the MD) displays pronounced oscillations due to the finite size of the box. These oscillations, which are indicative of coherent phonon modes, are less pronounced for smaller boxes, and are damped at longer times.

In order to compare the final sample temperature given by the MD to that predicted by the analytic model, the sample was allowed to thermalize for $100 \mathrm{ps}$ after the start of the ramp. The mean and standard deviation of the temperature over the final $5 \mathrm{ps}$ of the run were calculated for a number of
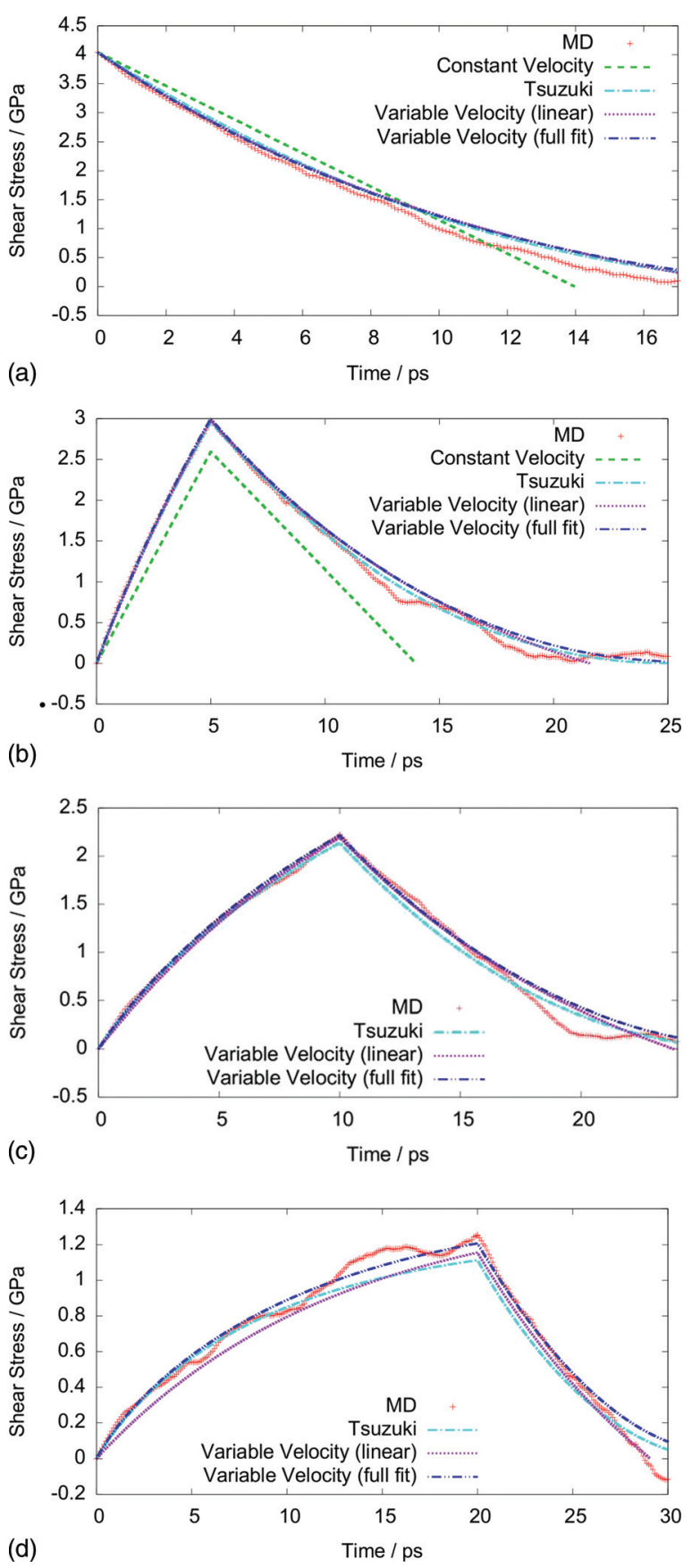

455

456

457

458

459

460

461

462

463

464

465

FIG. 3. (Color online) Shear relaxation as a function of time for different $t_{\text {rise }}$. Agreement between the model using a variable velocity and MD is extremely good, especially in the early stages of loading.

shear strain rates. These values are plotted against the theoretical temperature rise in Fig. 5.

The strong dependence of the temperature rise with ramp time is clearly seen, with excellent agreement between the MD and analytical models. It is clear that the temperature rise reaches its asymptotic value for strain rates greater than approximately $4 \times 10^{11} \mathrm{~s}^{-1}$, or ramp times of $250 \mathrm{fs}$. It is interesting to note that this time-scale is close to the value of the highest phonon frequencies in copper. ${ }^{47}$ This may well be indicative of a more general notion; that the phonon period describes the ultimate time-scale of shock processes in solids. 


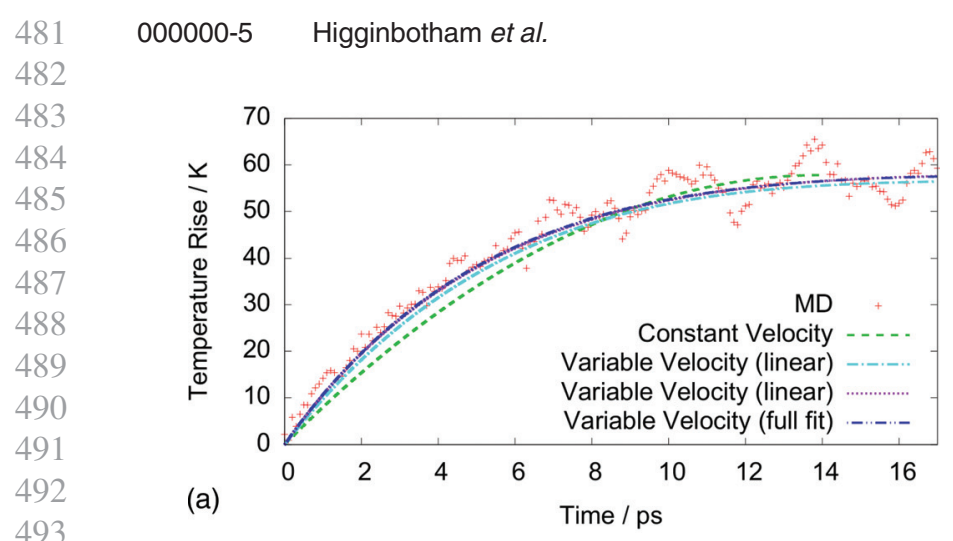

493

494

495

496

497

498

499

500

501

502

503

504

505

506

507

508

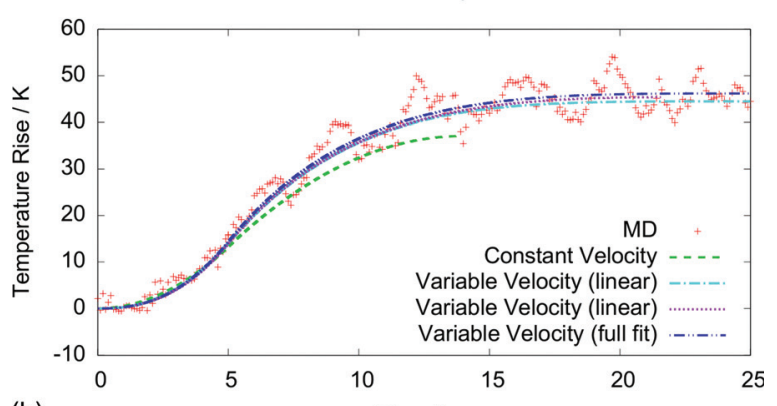

(b)
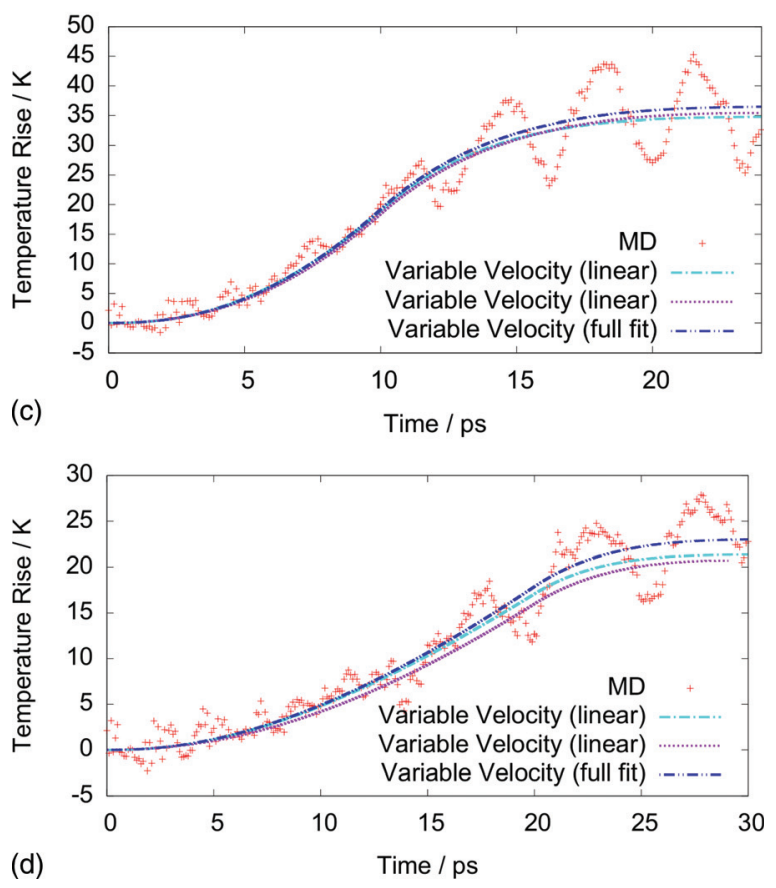

FIG. 4. (Color online) Temperature rise as a function of time for various shear strain rise-times.

Final temperatures derived by employing Tsuzuki's steady state dislocation velocities are seen to be consistently low. However, agreement is still within around $2 \mathrm{~K}$ over the entire range of strain rates examined. Although this suggests that steady state velocities may not be the ideal choice for this application, their relative abundance in the literature, coupled with the close agreement with dynamic simulations, makes their use attractive in determining temperature rise due to plastic work in ramps.

At the lowest strain rates studied, the constant velocity model fails, as expected. However, the relatively crude full fit still works well for temperature rises as low as one tenth of the asymptotic value, corresponding to shear strain rates of below $2 \times 10^{9} \mathrm{~s}^{-1}$, and ramp rise times of $50 \mathrm{ps}$.

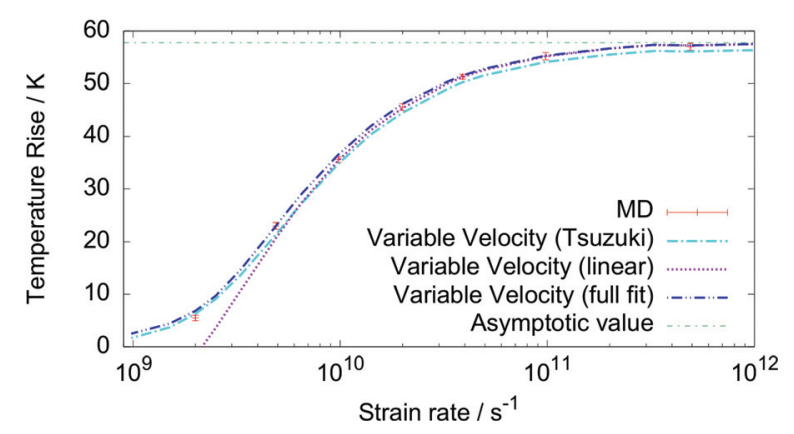

FIG. 5. (Color online) Temperature rise versus strain rate for both variable velocity models compared with MD. The asymptotic value is the temperature rise seen in a shock $\left(t_{\text {rise }}=0 \mathrm{ps}\right)$.

\section{SUMMARY AND CONCLUSIONS}

We have shown that for a typical fcc metal, simple models of plastic heating and shear relaxation agree extremely well with molecular dynamic simulations, provided that an appropriate stress-dependent velocity is used for the dislocation motion and that the dislocation density is known. We show that a simple linear velocity fit works well for higher strain rates, but modifications are required to accurately describe shear-strain ramps on longer time-scales, where dislocation flow occurs at lower shear stresses. Steady state dislocation velocities have also been shown to be sufficiently representative of dynamic behavior for this application. However, knowledge of the details of dislocation motion are not required to accurately predict the heating in this idealised sample.

For our system we show that a ramp of $t_{\text {rise }}=50$ ps or longer leads to significantly less plastic heating than a shock $\left(t_{\text {rise }}=0 \mathrm{ps}\right)$. This may have important implications for the creation of high pressure solids via laser compression where the strain rates and pressures attained are comparable to those accessed by MD simulations. However, in order to fully address this topic, the model must be generalized to address a number of issues.

For loading along arbitrary directions, including uniaxial compression along the principal crystallographic directions, the full tensor equations relating stress and strain have to be used, and the appropriate compliances have to be calculated. We must also take in to account the existence of multiple active slip planes in a plastically deforming solid. However, the route to implement these improvements is clear.

A more complex problem is the need to include terms for creation and destruction of dislocations. For instance, homogenous nucleation of dislocations ${ }^{47}$ or activation of Frank-Read type dislocation sources, ${ }^{2,4}$ could increase dislocation density. On the other hand, dynamic recovery, partly due to heating, would decrease dislocation density. Compliance may also change due to production or destruction of dislocations. These source terms need to be coupled to a model for dislocation mobility at high dislocation densities, when pinning will play a critical role. This could be solved using an effective drag coefficient, much smaller than the one used for the perfect single crystal. Note that experimental values of the drag coefficient ${ }^{48}$ are significantly smaller than those found in MD simulations, likely because of this effect. Cross-slip might also play a role for long rise times and large dislocation densities. ${ }^{49}$ 
We have considered here edge dislocations, but motion of screw dislocations should follow similar laws. ${ }^{50}$ However, the scenario might be more complex for materials which are not fcc. For instance bcc metals display extensive climb and extreme changes in the nature of dislocation motion at high strain rate, resulting in production of debris and extended twinning. $^{28}$

Despite these caveats, it may be feasible to carry out a reasonable prediction of plastic relaxation and plastic heating in fcc metals, without the need to carry out costly MD simulations of ramp loading.

\section{ACKNOWLEDGMENTS}

A.H. and M.S. are grateful for support from AWE. E.B. and J.S.W. gratefully acknowledge support from the Royal Society.

${ }^{1}$ G. I. Taylor, "The mechanism of plastic deformation of crystals. Part i. Theoretical," Proceedings of the Royal Society of London. Series A, Containing Papers of a Mathematical and Physical Character 145, 362 (1934), ISSN 09501207.

${ }^{2}$ J. S. Koehler, Phys. Rev. 86, 52 (1952).

${ }^{3}$ J. Nicholas, Acta Metallurgica 7, 544 (1959).

${ }^{4}$ M. A. Shehadeh, H. M. Zbib, and D. D. L. Rubia, International Journal of Plasticity 21, 2369 (2005).

${ }^{5}$ M. A. Meyers, Dynamic Behavior of Materials (Wiley, 1994).

${ }^{6}$ A. L. Ruoff, H. Xia, H. Luo, and Y. K. Vohra, Rev. Sci. Instr. 61, 3830 (1990).

${ }^{7}$ J. Edwards, K. T. Lorenz, B. A. Remington, S. Pollaine, J. Colvin, D. Braun, B. F. Lasinski, D. Reisman, J. M. McNaney, J. A. Greenough, R. Wallace, H. Louis, and D. Kalantar, Phys. Rev. Lett. 92, 075002 (2004).

${ }^{8}$ B. Yaakobi, T. R. Boehly, T. C. Sangster, D. D. Meyerhofer, B. A. Remington, P. G. Allen, S. M. Pollaine, H. E. Lorenzana, K. T. Lorenz, and J. A. Hawreliak, Phy. Plasmas 15, 062703 (2008).

${ }^{9}$ R. F. Smith, J. H. Eggert, A. Jankowski, P. M. Celliers, M. J. Edwards, Y. M. Gupta, J. R. Asay, and G. W. Collins, Phys. Rev. Lett. 98, 065701 (2007).

${ }^{10} \mathrm{C}$. A. Hall, Isentropic compression experiments on the sandia z accelerator, The 41st Annual Meeting of the Division of Plasma Physics of the American Physical Society 7, 2069 (2000).

${ }^{11}$ V. A. Tsukermann and A. I. Andeenko, Zh. Tekh. Fiz. 12, 185 (1942).

${ }^{12}$ L. V. Al'tshuler, Zh. Eksperim i. Teor. Fiz. 13, 11 (1943).

${ }^{13}$ Q. Johnson, A. Mitchell, R. N. Keeler, and L. Evans, Phys. Rev. Lett. 25, 1099 (1970).

${ }^{14}$ P. A. Rigg and Y. M. Gupta, Appl. Phys. Lett. 73, 1655 (1998).

${ }^{15}$ J. S. Wark, R. R. Whitlock, A. A. Hauer, J. E. Swain, and P. J. Solone, Phys. Rev. B 40, 5705 (1989).

${ }^{16}$ D. H. Kalantar, J. F. Belak, G. W. Collins, J. D. Colvin, H. M. Davies, J. H. Eggert, T. C. Germann, J. Hawreliak, B. L. Holian, K. Kadau et al., Phys. Rev. Lett. 95, 075502 (2005).

${ }^{17}$ D. K. Bradley, J. H. Eggert, R. F. Smith, S. T. Prisbrey, D. G. Hicks, D. G. Braun, J. Biener, A. V. Hamza, R. E. Rudd, and G. W. Collins, Phys. Rev. Lett. 102, 075503 (2009).

${ }^{18}$ B. L. Holian and P. S. Lomdahl, Science 280, 2085 (1998), http:// www.sciencemag.org/cgi/reprint/280/5372/2085.pdf.

${ }^{19}$ T. C. Germann, B. L. Holian, P. Lomdahl, and R. Ravelo, Phys. Rev. Lett. 84, 5351 (2000).
${ }^{20}$ E. M. Bringa, J. U. Cazamias, P. Erhart, J. Stölken, N. Tanushev, B. D. Wirth, R. E. Rudd, and M. J. Caturla, J. Appl. Phys. 96, 3793 (2004).

${ }^{21}$ E. M. Bringa, K. Rosolankova, R. E. Rudd, B. A. Remington, J. S. Wark, M. Duchaineau, D. H. Kalantar, J. Hawreliak, and J. F. Belak, Nat. Mater. 5, 805 (2006)

${ }^{22}$ D. Mordehai, I. Kelson, and G. Makov, Phys. Rev. B 74, 184115 (2006).

${ }^{23}$ D. Mordehai, Y. Ashkenazy, I. Kelson, and G. Makov, Phys. Rev. B 67, 024112 (2003).

${ }^{24}$ E. Bitzek and P. Gumbsch, Mat. Sci. Eng. A 387, 11 (2004).

${ }^{25}$ E. Bitzek and P. Gumbsch, Mat. Sci. Eng. A 400, 40 (2005).

${ }^{26}$ H. Tsuzuki, P. S. Branicio, and J. P. Rino, Appl. Phys. Lett. 92 (2008).

${ }^{27}$ H. Tsuzuki, P. S. Branicio, and J. P. Rino, Acta Materialia 57, 18431855 (2009).

${ }^{28}$ J. Marian, W. Cai, and V. V. Bulatov, Nat. Mater. 3, 158 (2004).

${ }^{29}$ J. A. Y. Vandersall and B. D. Wirth, Philosophical Magazine 84, 3755 (2002).

${ }^{30}$ H. M. Zbib and T. D. de la Rubia, Int. J. Plastic 18, 1133 (2002).

${ }^{31}$ A. Arsenlis, W. Cai, M. Tang, M. Rhee, T. Oppelstrup, G. Hommes, T. G. Pierce, and V. V. Bulatov, Modelling and Simulation in Materials Science and Engineering 15, 553 (2007).

${ }^{32}$ M. Shehadeh, E. Bringa, H. Zbib, J. M. McNaney, and B. A. Remington, Appl. Phys. Lett. 89, 171918 (2006).

${ }^{33}$ W. J. Murphy, A. Higginbotham, J. S. Wark, and N. Park, Phys. Rev. B. 78, 014109 (2008).

${ }^{34}$ P. K. W. Planteon, and D. Francke, Computational Materials Science 7, 75 (1996).

${ }^{35}$ R. Armstrong, C. Coffey, and W. Elban, Acta Metall. 30, 2111 (1982), ISSN 0001-6160.

${ }^{36} \mathrm{M}$. A. Meyers and K. K. Chawla, Mechanical Behaviors of Materials (Prentice Hall, Inc., 1999) pp. 570-580.

${ }^{37}$ G. Ravichandran, A. J. Rosakis, J. Hodowany, and P. Rosakis, CP620, Shock Compression of Condensed Matter (2001), edited by M. D. Furnish, N. N. Thadhani, and Y. Horie.

${ }^{38}$ S. Plimpton, J. Comp. Phys. 117, 1 (1995).

${ }^{39}$ Y. Mishin, M. J. Mehl, D. A. Papaconstantopoulos, A. F. Voter, and J. D. Kress, Phys. Rev. B. 63, 224106 (2001).

${ }^{40}$ J. Chang, W. Cai, V. Bulatov, and S. Yip, Mat. Sci.Eng. A 309, 160 (2001).

${ }^{41}$ W. J. Murphy, A. Higginbotham, G. Kimminau, B. Barbrel, E. M. Bringa, J. Hawreliak, R. Kodama, M. Koenig, W. McBarron, M. A. Meyers, B. Nagler, N. Ozaki, N. Park, B. Remington, S. Rothman, S. M. Vinko, T. Whitcher, and J. S. Wark, Condensed Matter 22, 065404 (2010).

${ }^{42}$ This non-zero pressure is a result of the finite box size. Simulations carried out in a fully relaxed sample showed no quantitative differences in shear stress relaxation or temperature rise within the error bars.

${ }^{43}$ This constant compliance was determined by both straining the system without allowing relaxation, and by monitoring the stress and strain during dynamic relaxation of a sample from $4 \mathrm{GPa}$ shear stress. This dynamic calculation was achieved by monitoring dislocation displacement, and thus plastic strain, as described in Sec. III B. In both cases linearity was observed over the entire range of stresses discussed in this paper.

${ }^{44}$ J. Marian and A. Caro, Phys. Rev. B 74, 024113 (2006).

${ }^{45}$ C. L. Kelchner, S. J. Plimpton, and J. C. Hamilton, Phys. Rev. B 58, 11085 (1998).

${ }^{46}$ D. L. Olmsted, L. G. Hector, Jr, W. A. Curtin, and R. J. Clifton, Modelling and Simulation in Materials Science and Engineering 13, 371 (2005).

${ }^{47}$ G. Kimminau, P. Erhart, E. M. Bringa, B. Remington, and J. S. Wark, Phys. Rev. 81, 092102 (2010).

${ }^{48}$ D. Hull and D. Bacon, Introduction to Dislocations 4th Ed. (ButterworthHeinemann, 2001).

${ }^{49}$ D. Mordehai, I. Kelson, and G. Makov, Mat. Sci. Eng. A. 400401, 37 (2005).

${ }^{50}$ Z. Q. Wang and I. J. Beyerlein, Phys. Rev. B. 77, 184112 (2008). 
AQ1: Please define NVE

AQ2: Please reword Fig. 1 without color words, so that the figure will be understood by readers of the black-and-white print version.

AQ3: Please provide DOI number for Refs. 11 and 12.

AQ4: Please supply full list of authors for Ref. 16 if possible. 